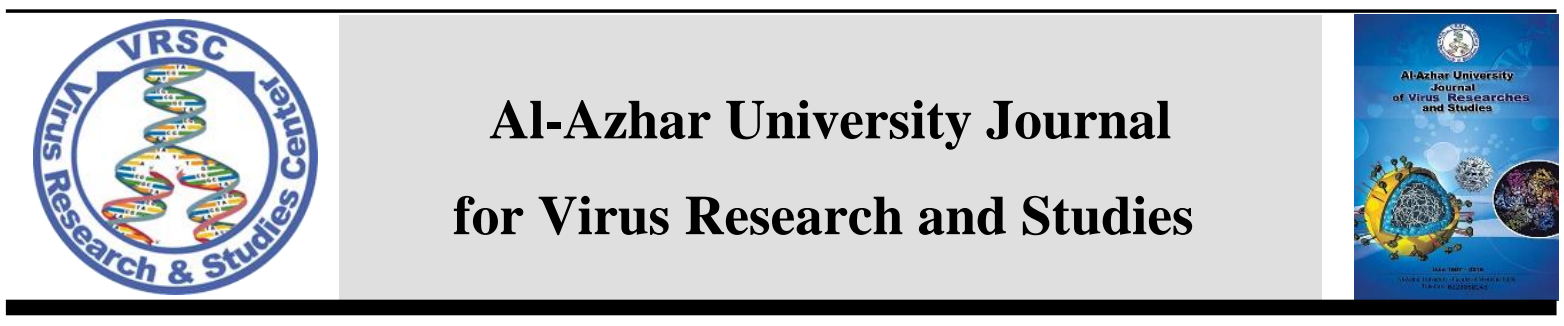

\title{
Fasciola Gigantica: Tegumental Surface Alterations Following Treatment in Vitro with Plumbagin
}

\author{
Doaa Abdel Fattah ${ }^{1}$, Khaled A. Temsah*2, Walaa A. S. El Kholy ${ }^{1}$, Amal A. El Kholy³, \\ Marwa A. El-Dardiry ${ }^{4}$ \\ ${ }^{1}$ Department of Parasitology, Faculty of Medicine, Al-Azhar University, Egypt. \\ ${ }^{2}$ Department of Parasitology, Damietta Faculty of Medicine, Al-Azhar University, \\ Egypt.
}

${ }^{3}$ Department of Clinical Pharmacy, Faculty of Medicine, Ain Shams University, Egypt.

${ }^{4}$ Department of Medical Parasitology, Faculty of Medicine, Fayoum University, Egypt.

*E-mail: drkhaled2008@yahoo.com

\begin{abstract}
Fascioliasis is a parasitic disease caused by liver fluke species of the genus Fasciola. Triclabendazole is the only known treatment to be efficient against both the pre- adults and adults' worms in hepatic parenchyma and the bile ducts, respectively, however, its effectiveness was found to be confronted by some resistance issues. This work aimed to investigate the antiparasitic effect of Plumbagin (PB) against the adult Fasciola gigantica $(F$. gigantica) parasite. PB is natural yellowish quinonoid compound, 5-hydroxy-2-methyl-1,4naphthoquinone, which is known to have an antiparasitic and anthelmintic activity in both humans and animals. In the current study, the anthelmintic efficacy of PB was assessed by using 40 flukes of the adult $F$. gigantica which were divided into four groups (10 each). Three groups were treated with different concentrations of PB $(1,10$, and $100 \mu \mathrm{g} / \mathrm{ml})$ and the fourth group was left untreated to act as a negative control. The efficacy of PB against $F$. gigantica was assessed by scanning electron microscopic (SEM) and histopathological examination of the tegument area in the posterior part of the fluke's body. The results showed that PB caused tegumental alterations, including swelling, blebbing, and rupture of the tegument, loss of spines, eventual erosion, and even desquamation of the total tegument, in a dose dependentmanner. The severity of tegumental damage increased, proportionally, with the concentrations of $\mathrm{PB}$ to indicate its fasciolicidal effect against adult $F$. gigantica. Further studies are recommended for its potential use as an anthelmintic against Fasciola sp. infections.
\end{abstract}

Keywords: Fasciola gigantica; Anthelmintic; Plumbagin; Tegumental morphological changes.

\section{Introduction}

Fascioliasis is a parasitic disease caused by liver fluke species of the genus Fasciola (Digenea: Fasciolidae) and considered as one of the most important helminthic diseases. The giant hepatic flukes, $F$. gigantica and $F$. hepatica are plant-borne 
trematodes, leaf-shaped flatworms, which were diagnosed in the liver of some economical livestock such as cattle, sheep, buffaloes, goats, swine, and other domestic animals while the world health organization (WHO) had reported their significant impact on the human being, as well [1]. Both species had significant impacts on the growth rate, development, and productivity of ruminants, therefore, they are considered economically substantial [2]. Both species are widely distributed worldwide, where the F. hepatica is commonly distributed in the urban areas which are characterized as high temperate zones and the $F$. gigantica which is distributed in tropical zones, such as Africa and Asia [3]. Most of the previous reports have focused on $F$. hepatica, whereas fewer studies have been conducted on $F$. gigantica. The treatment strategy for fascioliasis depends, exclusively, on the usage of different effective anthelminthic drugs [4]. Since 1983, the benzamide compound 'Triclabendazole' is the only approved veterinary treatment of Fascioliasis and even for the human infections, that was recommended by the WHO in 1997, especially for the endemic countries of the high fascioliasis burden [5]. Triclabendazole is the only known drug to be efficacious against the pre-adult and adult worms of $F$. gigantica in both the hepatic parenchyma and the bile ducts, respectively, despite of the drug resistance that was discussed in several studies [6,7]. The risk of resistance elevation to triclabendazole is due to either the longterm usage for the livestock treatment, particularly in the highly endemic areas; the availability and wide distribution of the drug, worldwide, which encourages its continuous and direct usage for the treatment of Fascioliasis, or the lack of awareness of the resistance issues as it was reported recently in some of the developing countries [8]. Currently, the need of new effective alternative treatment for fascioliasis became highly essential where species are becoming more and more resistant beside the universal resurgence for the replacement of synthetic drugs with other natural alternative therapies because of their higher biosafety level and fewer side effects [4].

Plumbagin (PB, 5-hydroxy-2-methyl-1,4naphthoquinone) is a natural yellowish quinonoid compound that is isolated from the roots of many plants such as the subscandent shrubs of Chitrak (Plumbago zeylanica) that was previously used by the traditional medicine for treatments of the symptoms of diarrhea, anasarca, and skin disease or as an appetizer and antiflatulence, as well [9]. Many pharmaceutical characteristics had been previously reported for $\mathrm{PB}$ such as the antioxidant [10], antimicrobial [11], antiinflammatory [12], and antineoplastic effects [13]. The antiparasitic activity of PB was reported for the first time by Atjanasuppat and colleagues in 2009, besides, they suggested that PB might possess an anthelmintic effect against Schistosomes, as well, in both humans and animals [14]. In cancer research, PB was found to suppress the expression of the nuclear factor kappa-light-chain-enhancer of activated $B$ cells (NF- $\kappa \mathrm{B})$ which contributed to the regulation of different essential cellular processes such as proliferation, immunoregulatory genes expression, and apoptosis which are involved in the both the innate and adaptive immune responses [10]. Moreover, PB affects the signaling of the redox reaction that induces the accumulation of the reactive oxygen species (ROS), besides, its ability to react with trace metals present in the biological system [14]. The adult helminthes infected the human receive, commonly, less or no amount of oxygen which develops a special type of anaerobic metabolism mediated by NADH-fumarate reductase and the rhodoquinone instead of ubiquinone (CoQ) [15]. Previous studies showed that PB had a significant antiparasitic effect against multiple parasite species such as Schistosoma 
mansoni [16], Leishmania sp. [17], and Plasmodium berghei infected malaria [18], as it can act as electrons acceptor that competes with the CoQ in the electron transport chain [19]. Because of the few reported studies about the antiparasitic effect of $\mathrm{PB}$ against $F$. gigantica. In the current study, the anthelmintic effect of PB against adult $F$. gigantica was evaluated by estimating the tegumental surface alterations in the treated parasites, in vitro.

\section{Materials and Methods}

\subsection{Parasites:}

All animal experiments were performed under the guidelines of the Egyptian Institutional Animal Care and Use Committee (IACUC) https://www.aalas.org/iacuc and the ethical guidelines of the Theodor Bilharz Research Institute (TBRI), Giza, Egypt. The adult liver flukes F. gigantica were collected from either the bile ducts or the gall bladders of animals condemned at AlBasateen slaughterhouse in Cairo, Egypt. They were washed several times with $0.85 \% \mathrm{NaCl}$ solution and only intact and actively mobile flukes were selected for the study.

\subsection{Drug preparation:}

The stock solution was prepared by mixing one gram of the purified PB extract with five $\mathrm{ml}$ of dimethylsulphoxide (DMSO), then the volume was completed to one liter by distilled water [14]. The sterile RPMI1640 (Caisson Laboratories Inc. Smithfield, UT, USA) supplemented with L-glutamine, penicillin (50 IU/ml), streptomycin $(50 \mu \mathrm{g} / \mathrm{ml})$, and gentamycin (30 IU/ml) (Sigma-Aldrich, St. Louis, Missouri, USA) was used for the in vitro incubation of worms. The working solutions of PB $(1,10$, and $100 \mu \mathrm{g} / \mathrm{ml})$ were prepared by dissolving in RPMI-1640 complete medium as previously described [11].

\subsection{Study design:}

The adults $F$. gigantica were recovered under sterile conditions in a laminar flow cabinet. The worms were kept in a petri dish with the complete medium. They were divided into four groups (ten flukes each) classified as; Group 1 (negative control) where the worms were incubated in the complete medium containing $0.1 \%$ DMSO, Group 2, 3, and 4 where flukes were incubated in the complete medium containing $1 \mu \mathrm{g} / \mathrm{ml}$, and $100 \mu \mathrm{g} / \mathrm{ml}$ of $\mathrm{PB}$, respectively, for 24 hours at room temperature [20]. On the day of the examination, the flukes were collected, and their bodies were cut into two parts where the posterior two-third parts were used for the histopathological examination and the other anterior third parts were used for the scanning electron microscopy (SEM).

\subsection{Histopathological examination:}

As been described before by (Saowakon et al., 2009) [21] the posterior two-thirds of the worms were longitudinally oriented on a filter paper and fixed in $10 \%$ formalin. Later, the tissues were processed for paraffin embedding and sliced into microsections of $5 \mu \mathrm{m}$ thickness. These paraffin- embedded sections were dewaxed in xylene for two minutes, hydrated in serial descending grades of ethanol $(100 \%$, $90 \%, 70 \%$, and $50 \%$ ) for another two minutes each, and then washed with distilled water for two minutes, as well. Later, the sections were stained with hematoxylin $(\mathrm{H})$ and Eosin $(\mathrm{E})$. After staining, the sections were dehydrated in ascending grades of ethanol $(50 \%, 70 \%$, $90 \%$, and $100 \%$ ), followed by incubation with xylene and mounting by Canada balsam (Sigma-Aldrich, St. Louis, Missouri, USA). The sections were examined by Leica DMi1 inverted light microscope (Leica Microsystems Co., Wetzlar, Germany). 


\subsection{Scanning Electron Microscopic Examination:}

The anterior third parts were used for SEM examination as described by [22]. Briefly, samples were fixed in $2.5 \%$ buffered glutaraldehyde in 0.1 M PBS ( $\mathrm{pH} 7.4)$ at $4^{\circ} \mathrm{C}$ for 24 hours. Later the samples were washed with PBS and re-fixed with $1 \%$ osmic acid for one hour. The samples were washed again with PBS, dehydrated with ascending serial dilutions of ethyl alcohol (30, 50, 70, 90\%, and 100\%), and infiltrated with acetone. Later the samples were dried with SPI Critical Point Dryer and mounted in aluminum stubs, coated with gold in an SPI Module ${ }^{\mathrm{TM}} \mathrm{Vac} /$ Sputter coater, (SPI supplies, West Chester, PA, USA). Finally, the specimens were examined by the JSM-52500 LV scanning electron microscope (JEOL Ltd. Inc., Tokyo, Japan), in the "Electron Microscope Unit at the Faculty of Science, Al-Azhar University.

\section{Results}

\subsection{Normal morphology of the adult $F$. gigantica:}

In the current study, we scanned the normal surface morphological structures in the adult $F$. gigantica, group 1 , as shown in Fig.1, the gonopore was situated between the oral and ventral sucker while the entire surface of the tegument was covered by spines, except in the rims of both suckers, Fig.1A and Fig.1B. The size and shape of these spines were variable according to their location on the fluke surface as they were larger and tipped with finger-like protrusions on the anterior and middle areas, Fig. $1 \mathrm{C}$, while at the posterior and tail areas they looked smaller and the protrusions were reduced or even absent, Fig.1D. Furthermore, the tegumental surface appeared as a mesh-like network of tiny ridges and pits which became smoother at the rims of both suckers, Fig.1D. Remarkably, it was noticed that the number of spines was decreased, gradually, at the posterior parts of the body, Fig.1E.

\subsection{Histopathological examination:}

The effect of PB adult $F$. gigantica worm exposed tegument was assessed by the light microscopic examination, in a dosedependent matter, Fig.2. At the control group, the normal structure of the tegument (T) appeared smoothly and non-swelled with the spines (S) attached in the normal triangular shape where the dense $\mathrm{H} \& \mathrm{E}$ stain indicated that the spinal sockets were not evacuated, Fig.2A. At the lower dose of PB $(1 \mu \mathrm{g} / \mathrm{ml})$, the tegument was slightly swelled (ST), while some spines were either lost (LS) or appeared as sunken spines (SS), Fig.2B. The morphological alteration increased at the $\mathrm{PB}$ concentration of $10 \mu \mathrm{g} / \mathrm{ml}$ with an excessive swelling of the tegument and more sunken spines and lost (SS), Fig.2C, Fig.2D, \& Fig.2E. The severity of morphological changes was noticed at the PB dose of $100 \mu \mathrm{g} / \mathrm{ml}$, while the spines were completely lost and the tegumental syncytium was completely detached (DT) away, leaving an exposed basal lamina and empty spine sockets (ESS), Fig.2F.

\subsection{Scanning electron microscopic (SEM) examination:}

The SEM results showed no changes in the tegument surface of the anterior parts of the flukes incubated in RPMI-1640 medium supplemented $0.1 \%$ DMSO (Group 1). The Oral and ventral suckers appeared smooth and surrounded with thick rims which were covered with transverse folds, Fig.3. For the flukes of group 2 (Fig.4), the incubation with $1 \mu \mathrm{g} / \mathrm{ml}$ of $\mathrm{PB}$ caused light morphological changes represented by the slight swelling of both suckers, despite the ventral sucker appeared more swollen with swollen tegument and clear deep furrows between spines, Fig.4B \& Fig.4C. Some of the spines were sunken in the surrounding tegument with their tips protruding from a swollen base, Fig.4C. Other spines were 
dislodged and lost their finger-like protrusions while their sockets were exposed in the syncytium, Fig.4D. The concentration of $10 \mu \mathrm{g} / \mathrm{ml}$ of PB extract caused significant changes in the incubated flukes, Fig.5. The ventricle and the tegument were smoother and more swollen, Fig.5A \& Fig.5B. All the spines were, obviously, without their finger-like projections, so they appeared with smooth tips, Fig.5C \& Fig.5D. Some of the spines were dislodged and their sockets were exposed in the syncytium, Fig.5E \& Fig.5F. At the maximum concentrations of PB $(100 \mu \mathrm{g} / \mathrm{ml})$ the ventricles were severely damaged with abnormal smooth rims, Fig.6A \& Fig.6B. Most of the spines were completely destructed and dislodged from their places, exposing their empty spine sockets, Fig.6C \& Fig.6D. Moreover, suckers were severely destroyed, and the tegument was lost in some parts, Fig.6E.
A)

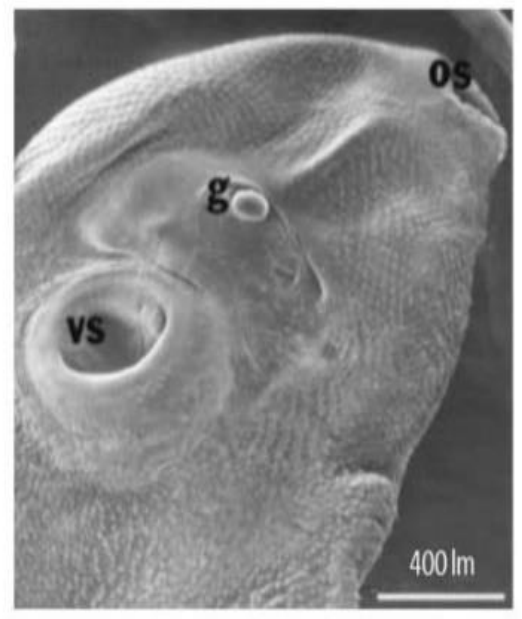

C)

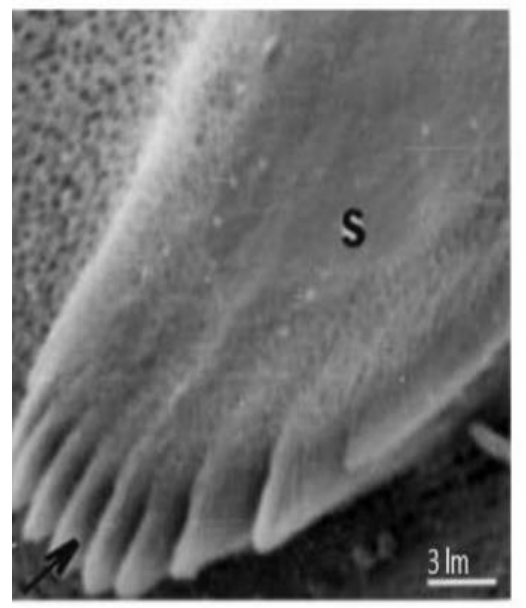

D)

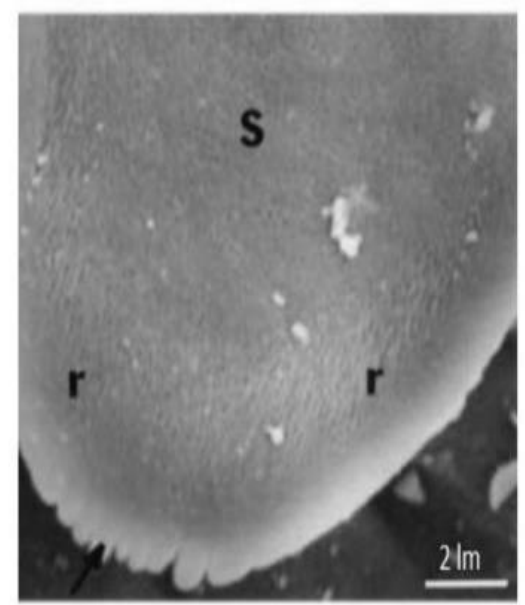

B)

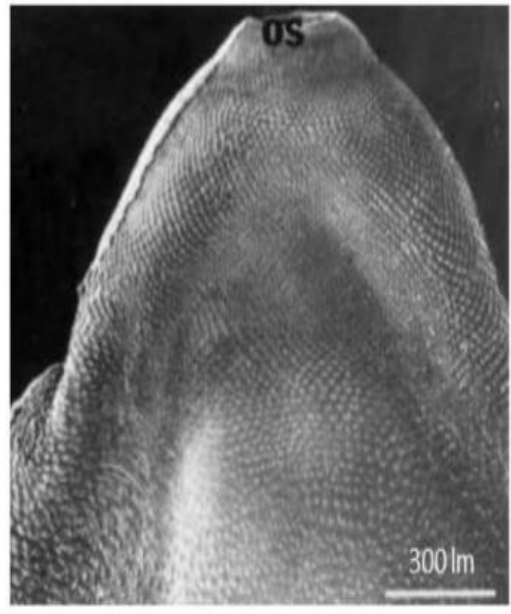

E)

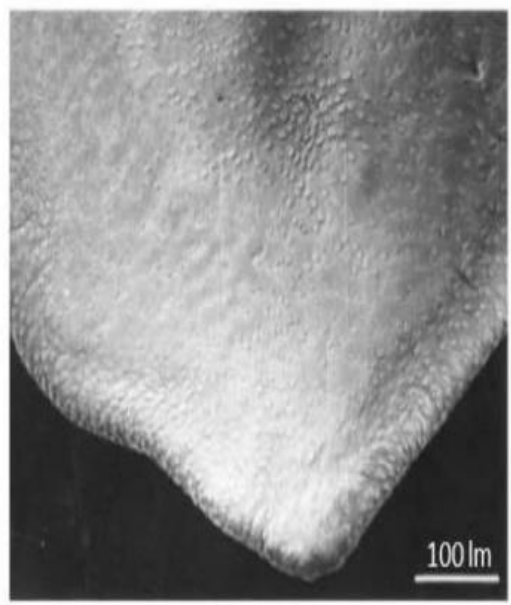

Figure (1): The Scanning electron micrographs of the control adult Fasciola gigantica. (A) Ventral surface of the apical cone showing the oral sucker (OS), ventral sucker (VS), and gonopore (g). The surface of the fluke is covered with spines, B) Dorsal surface of the apical cone region with the oral sucker (OS) at its anterior end, C) Spine (S) from the ventral surface of the apical cone region showing the numerous large finger-like projections (arrow) at its tip. D) Spine (S) from the dorsal surface of the anterior mid-body region of control adult $F$. gigantica showing fewer protrusions (arrow) at its tip than seen on spines from the anterior region. The surface of the tegument covering the spines takes the form of a meshwork of ridges and pits (r). E) Dorsal surface of the tail region of control adult $F$. gigantica showing a lower density of spines than seen in the anterior half of the fluke. 
A)
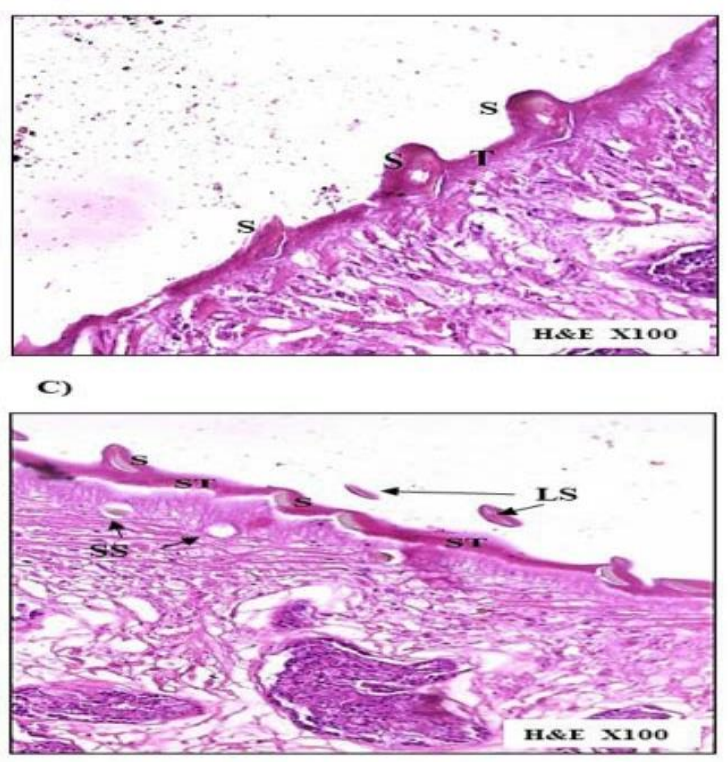

E)

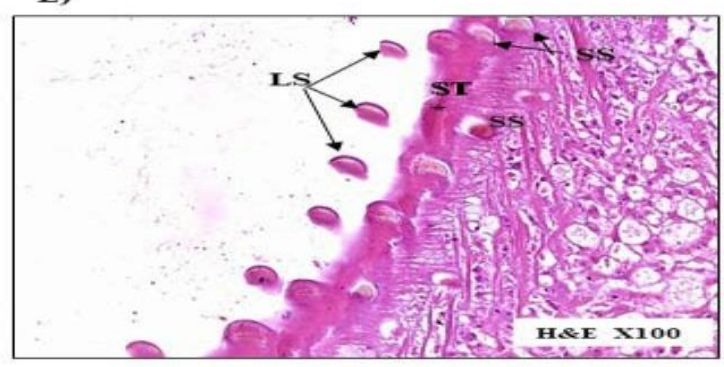

B)

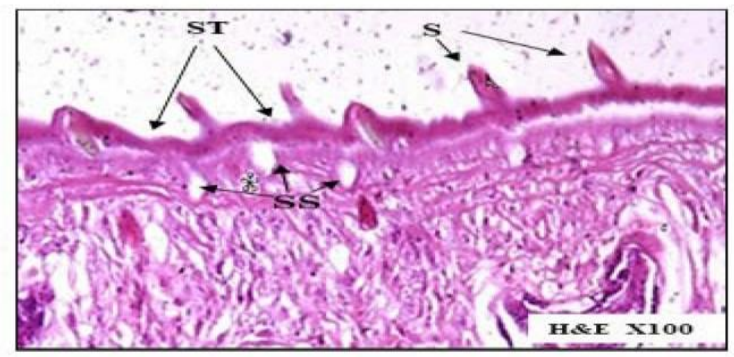

D)

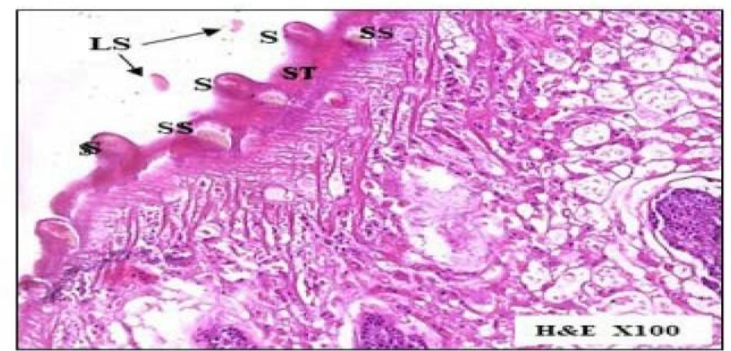

F)

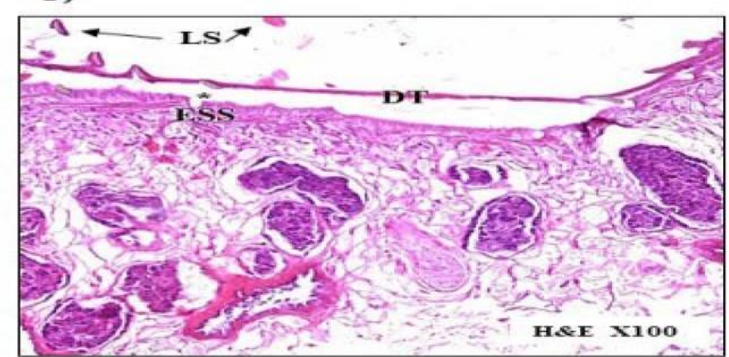

Figure (2): The Effect of Plumbagin on the tegument morphology of the adult Fasciola gigantica. Cross-sections of the tegument of the adult Fasciola gigantica stained with H\& E. A) The tegument was incubated with DMSO for 24 hours and showed normal tegument with an intact spine, B) The tegument was incubated with PB $(1 \mu \mathrm{g} / \mathrm{ml})$ for 24 hours and showed slightly Swollen Tegument (ST) with some Sunken Spines (SS) and Lost Spines (LS), C), D), E) The tegument was incubated with PB $(10 \mu \mathrm{g} / \mathrm{ml})$ for 24 hours and showed markedly swollen tegument with SS and LS. F) The tegument was incubated with PB $(100 \mu \mathrm{g} / \mathrm{ml})$ for 24 hours and showed the tegumental syncytium and spines were completely detached (DT) away, leaving an exposed basal lamina and empty spine sockets (ESS).

A)

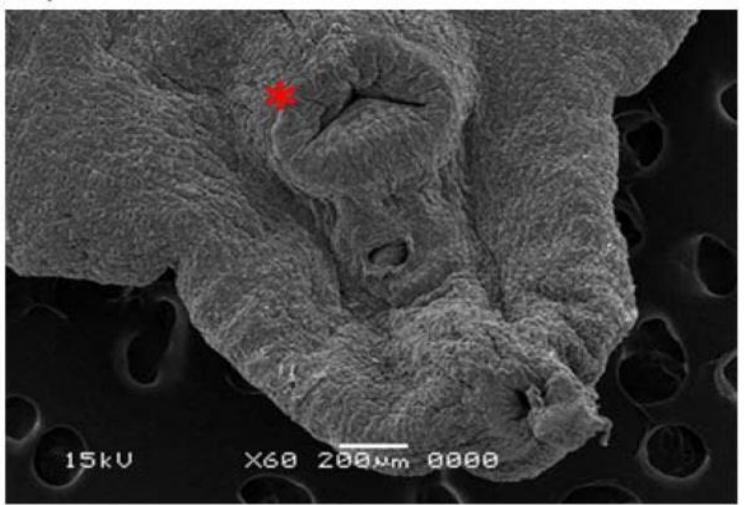

B)

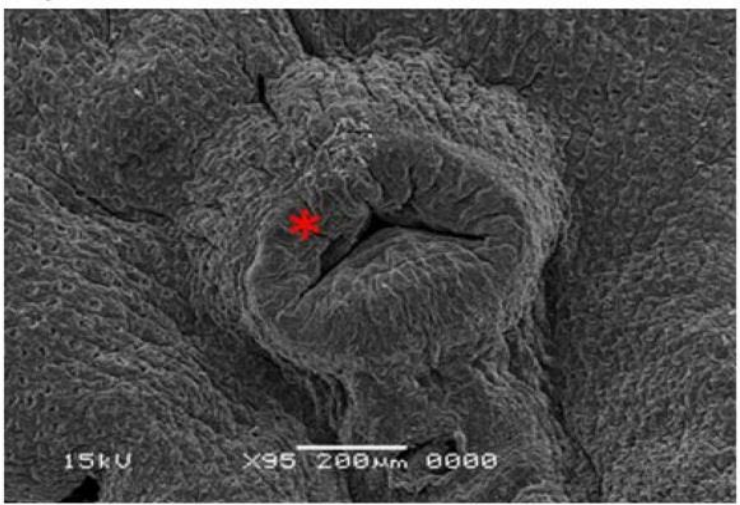

Figure (3): The morphology of the suckers in the tegument. A), B) The tegument surface of them flukes incubated in the medium containing $0.1 \%$ DMSO up to $24 \mathrm{~h}$ (Group 1) with oral and ventral suckers (red asterisk) appeared smooth with thick rims covered with transverse folds. The entire surface of the tegument was seen covered with spines, except for the rims of suckers 


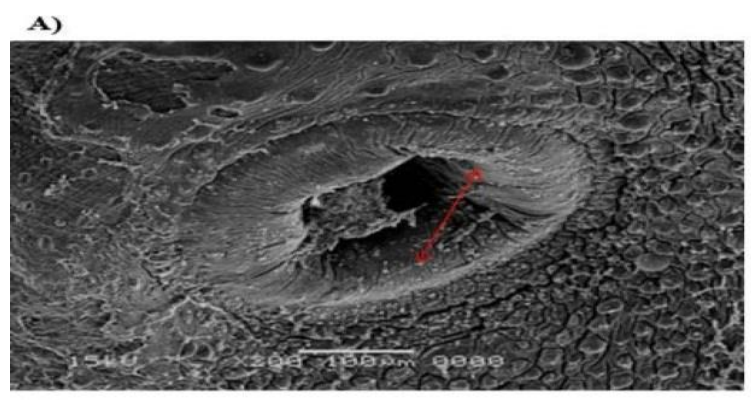

C)

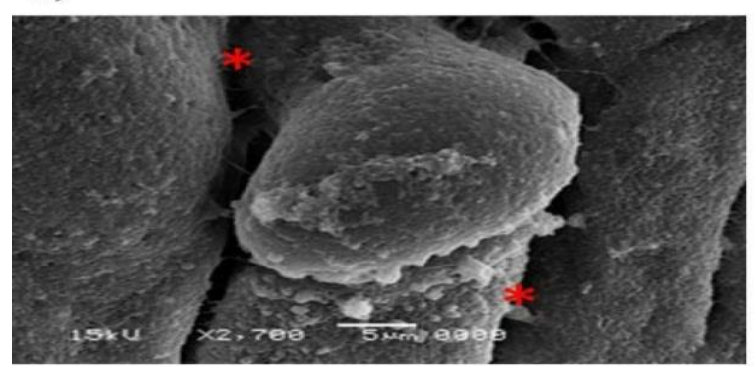

B)

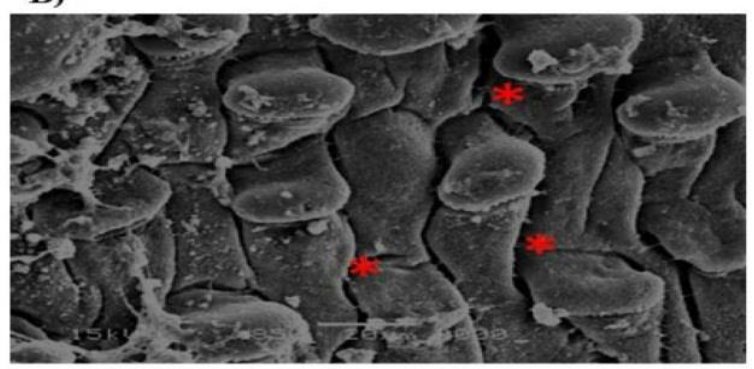

D)

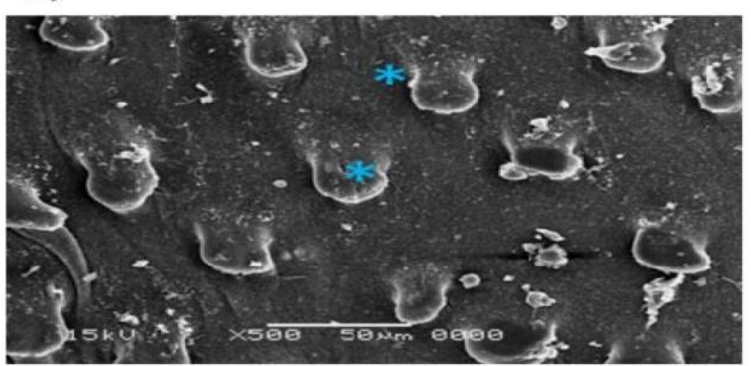

Figure (4): SEM of $F$. gigantica after 24 hours in vitro treatment with PB (1 $\mu \mathrm{g} / \mathrm{ml})$ (Group 2). A) The ventral sucker appeared more swollen (red arrow) than usual, B), C) The tegument below ventral sucker was found to be swollen with deep furrows (red asterisks) between spines, D) Some of the spines were dislodged (blue asterisks), exposing their sockets in the syncytium

A)

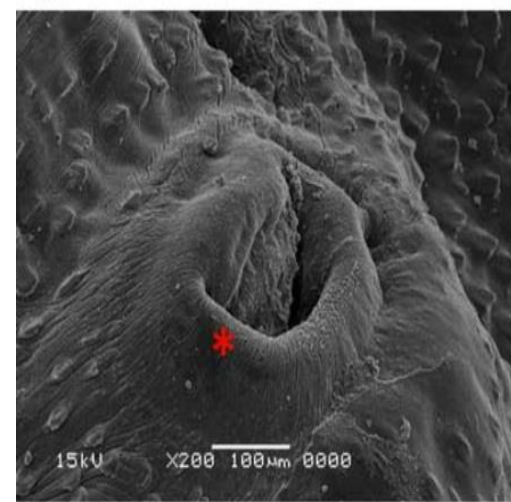

D)

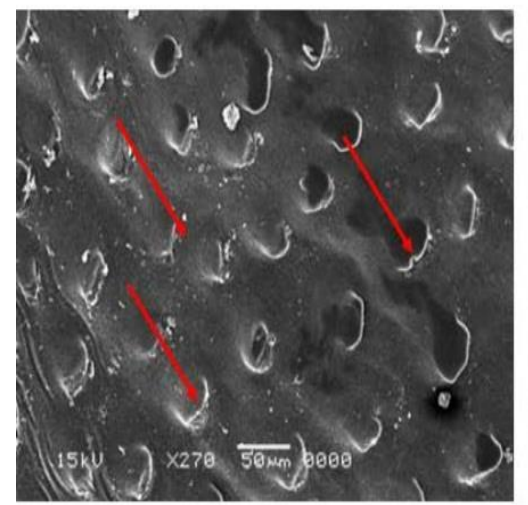

B)

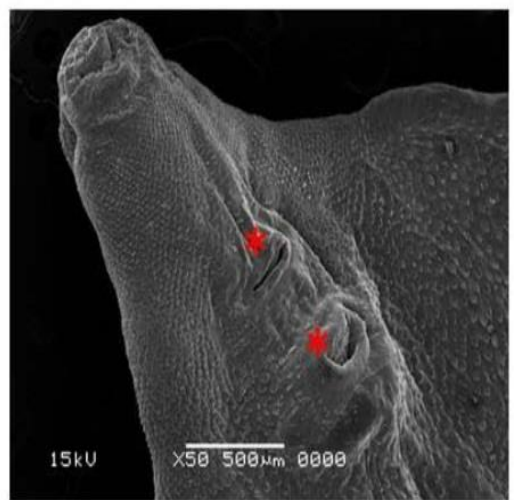

E)

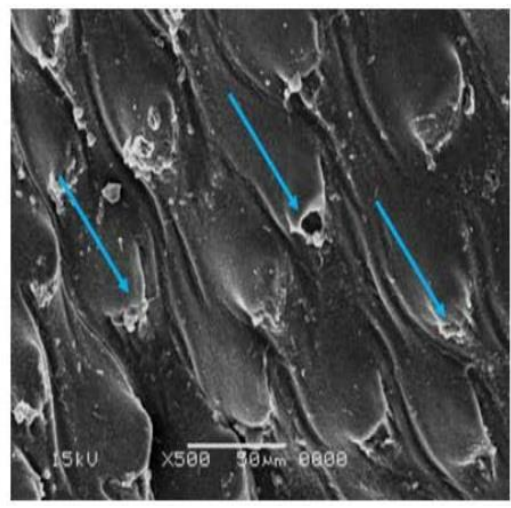

C)

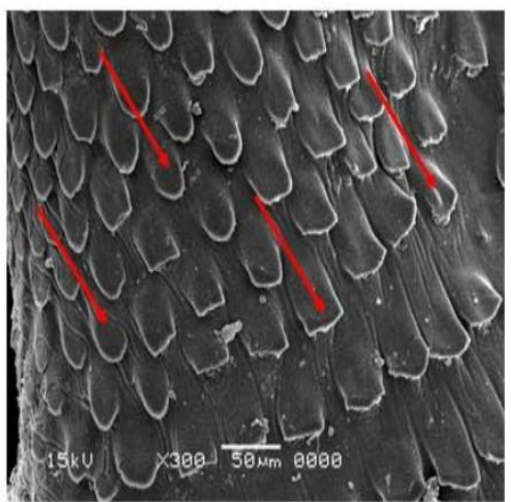

F)

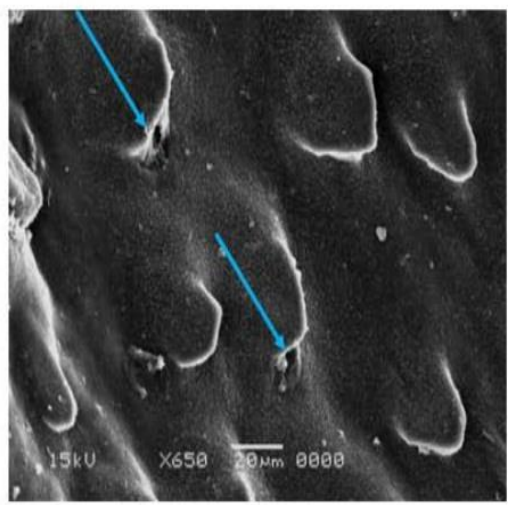

Figure (5): SEM of $F$. gigantica after 24 hours in vitro treatment with PB (10 $\mu \mathrm{g} / \mathrm{ml})$ (Group 3). A), B) The suckers (red asterisks) swelled and loss their ridges. C), D), E), F) All the spines lost their finger-like projections (red arrows) so they appeared with smooth tips and some of them were dislodged exposing their sockets in the syncytium (blue arrows). 
A)

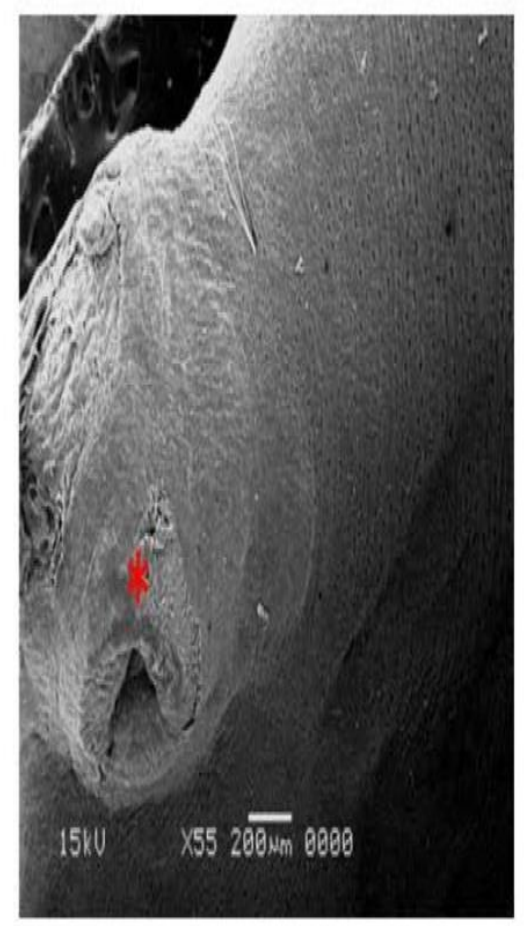

B)

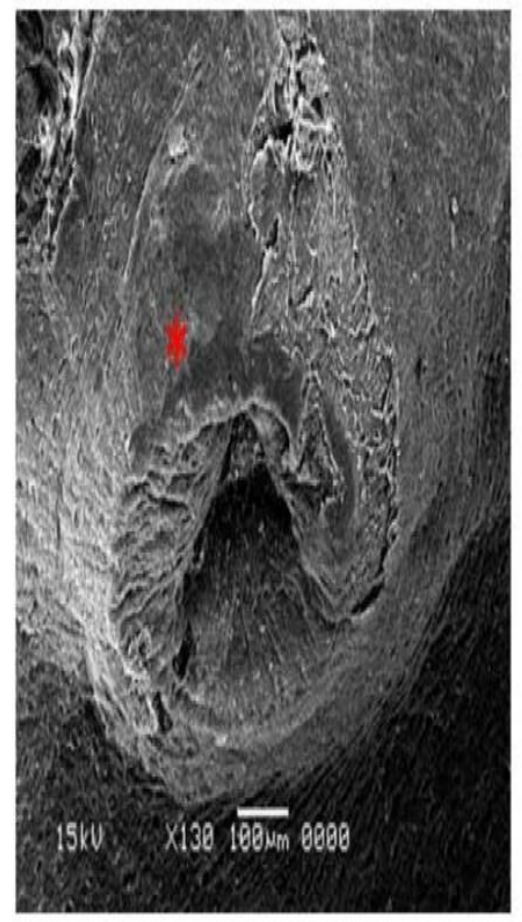

E)

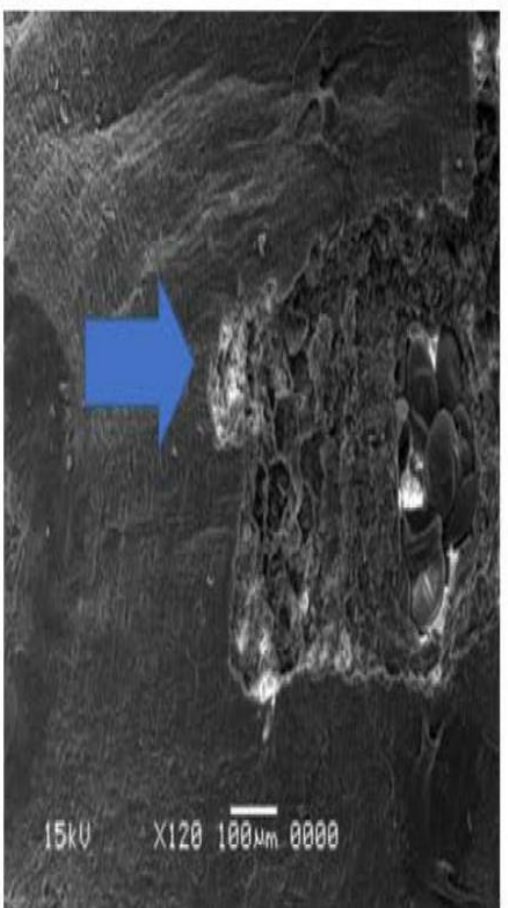

C)

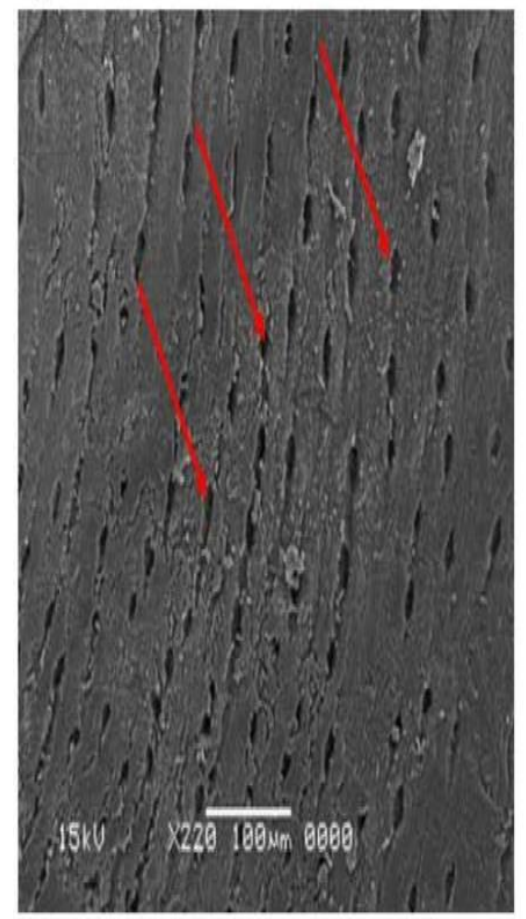

D)

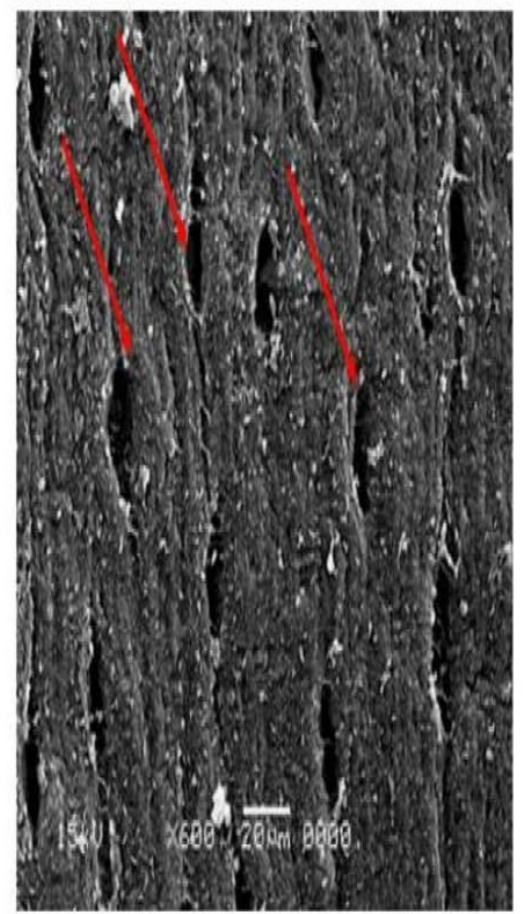




\section{Discussion}

In the current study, we isolated and tested the effect of PB in a dose-dependent manner on the adult $F$. gigantica. Two Fasciola species, $F$. hepatica and $F$. gigantica are symmetrically, dominant in the infected livestock in Egypt; therefore, it was indispensable to distinguish the normal morphological structure of $F$. gigantica flukes. The normal morphological structures of $F$. gigantica was described before [23], where they reported that the adult flukes were leaf-like worms with the length of almost $40 \mathrm{~mm}$ and tipped ends. The anterior end is characterized by a prominent apical cone containing the circular tipped oral sucker, where the posterior end was bounded by the ventral sucker with thick rims. The surface is roughly supplied by numerous, small, and closely spaced spines, where the tegumental surface between them is characterized with highly transverse folds and grooves which are further distinguished by a highly complicated network of ridges and variable-sized slits [23]. That was in agreement with the findings in the current study which approve the classification of the isolated species. The control of fascioliasis is deeply dependent on the use of chemical drugs [21], however, the excessive and continuous usage of these drugs had arisen a new era of drug resistance as had been reported in many endemic regions of the world $[24,25]$. The current trend in medical and pharmacological research, is the investigating of new treatments of natural origin, because of their higher biosafety levels and fewer side effects [24,26]. The morphological changes of the parasite surfaces have paid a lot of attention in recent studies as the development of the electron microscopy had facilitated the understanding of the complex morphological exchanges which is of great importance to the study of the host-parasite and drug- parasite interactions [27]. In the current study, we investigated the effect of
PB which is one of the important plant extracts in the medical studies, on the tegument of adult $F$. gigantica in a dosedependent manner. The results demonstrated a series of tegumental changes detected by both the histopathological and SEM examination. These changes occurred in a definite sequence, where the swelling was the first observed sign, followed by blebbing, then dislodgement of the spines with disruption of their covering tegument where the severity of changes increased with higher doses of PB. As been previously reported, the damage of the tegument is thought to induce even deeper alterations in the underlying tissues and internal organs which would, indeed, cause more damage to the whole parasite body [22], and disrupt many of the physiological processes associated with the tegument including osmoregulation, nutrient uptake, secretion, synthesis, and immune- protection [28]. So, no doubt, the destruction of spines and sensory papillae will deprive the parasite of its vital functions, facilitating its clearance. In agreement with our finding, a previous study showed that the anthelmintic efficacy and fasciolicidal activity of 1,10 and $100 \mu \mathrm{g} / \mathrm{ml}$ of PB that caused significant tegumental alterations in $F$. gigantica with significant swelling, blebbing, rupture, erosion, lesion, and desquamation of the tegument, where most of the spines were lost [29]. Similar studies had shown similar effects of other plant extracts on adult Fasciola tegument ultrastructure such as the effect of artesunate and artemether that showed a dose-dependent effect on in vitro cultured Fasciola adults where the swelling of tegument and complete loss of spines were reported leading to the fluke's death [30,31]. Another study showed that the aqueous extract of Artocarpus lakoocha on had similar morphological changes on the adult $F$. gigantica, including tegumental swelling and blebbing, besides the further osmotic imbalance induced by the secondary influx of $\mathrm{Na}+$ and water with consequent swelling of the syncytium [21]. 
Another study showed the in vitro fasciolicidal activity of Meryta denhamii plant extracts against adult Fasciola and showed tegumental changes similar to the above findings [32]. On the other hand, similar studies had reported the effect of PB on different parasitic species. The in vitro treatment of Schistosoma mansoni showed significant ultrastructural changes in the tegument including, swelling, blebbing, and loss of spines [33]. Another study showed that $\mathrm{PB}$ induced severe ultrastructural changes in the promastigote and amastigote forms of Leishmania donovani and induced apoptosis-like cell death [34] (Awasthi, et al.,2016).

\section{References}

1. Waikagul, J., Sato M., Sato, M.O., (2015). Foodborne trematodes (Chapter 10), Editor: Gajadhar A. A. In Woodhead Publishing Series in Food Science, Technology and Nutrition, Foodborne Parasites in the Food Supply Web.1st edition. Woodhead Publishing, pp 221- 255.

2. Beesley, N. J., Caminade, C., Charlier, J., et al., (2018). Fasciola and fasciolosis in ruminants in Europe: Identifying research needs. Transbound. Emerg. Dis. 65, 199216.

3. Phalee, A., Wongsawad, C., Rojanapaibul, A., Chai, J., (2015). Experimental Life History and Biological Characteristics of Fasciola gigantica (Digenea: Fasciolidae) Korean J. Parasitol. 53, 59-64.

4. Ullah, R., Rehman, A., Zafeer, M. F., et al., (2017). Anthelmintic Potential of Thymoquinone and Curcumin on Fasciola gigantica. PloS one. 12, e0171267.
In conclusion, the novel findings of this study indicated a high therapeutic potential of PB as an anti-fascioliasis drug. Multiple studies had demonstrated the fasciolicidal activity of PB as it caused severe structural changes for the adult $F$. gigantica tegument and other species. So, PB represents a suitable alteration for the control and treatment of fascioliasis instead of the current synthetic compounds which were reported to have serious side effects and lower safety levels. Further study on the efficiencies of PB treatment in vivo, as well as possible side effects to hosts, should be investigated.

5. McCarthy J.S., Moore, T. A., (2015). 42 - Drugs for Helminths, Editor(s): John E. Bennett, Raphael Dolin, Martin J. Blaser. In: Mandell, Douglas, and Bennett's Principles and Practice of Infectious Diseases. $8^{\text {th }}$ Edition. Content Repository Only! pp 519-527.e3.

6. Gandhi, P., Schmitt, E. K., Chen, C. W., Samantray, S., Venishetty, V. K., Hughes, D., (2019). Triclabendazole in the treatment of human fascioliasis: a review. T. Roy. Soc. Trop. Med. H. 113, 797-804.

7. Cabada, M. M., Lopez, M., Cruz, M., Delgado, J. R., Hill, V., White, A. C., Jr, (2016). Treatment Failure after Multiple Courses of Triclabendazole among Patients with Fascioliasis in Cusco, Peru: A Case Series. PLOS Negl. Trop. Dis. 10, e0004361.

8. Cwiklinski, K., O'Neill, S. M., Donnelly, S., Dalton, J. P., (2016). A prospective view of animal and human Fasciolosis. Parasite Immunol. 38, 558-568. 
9. Sand, J. M., Bin Hafeez, B., Jamal, M. S., et al., (2012). Plumbagin (5hydroxy-2-methyl-1,4naphthoquinone), isolated from Plumbago zeylanica, inhibits ultraviolet radiation-induced development of squamous cell carcinomas. Carcinogenesis. 33, 184 190.

10. Kuan-Hong, W., \& Bai-Zhou, L. (2018). Plumbagin protects against hydrogen peroxide- induced neurotoxicity by modulating NF- $\kappa \mathrm{B}$ and Nrf-2. Arch. Med. Sci. 14, 11121118

11. Nair, S.V., Baranwal, G., Chatterjee, M., et al., (2016). Antimicrobial activity of plumbagin, a naturally occurring naphthoquinone from Plumbago rosea, against Staphylococcus aureus and Candida albicans. Int. J. Med. Microbiol. Suppl. 306, 237-248.

12. Reese, S., Vidyasagar, A., Jacobson, L., et al., (2010). The Pin 1 inhibitor juglone attenuates kidney fibrogenesis via Pin 1-independent mechanisms in the unilateral ureteral occlusion model. Fibrogenesis Tissue Repair 3, 1.

13. Liu, Y., Cai, Y., He, C., Chen, M., Li, H., (2017). Anticancer Properties and Pharmaceutical Applications of Plumbagin: A Review. Am. J. Chinese. Med. 45, 423-441.

14. Atjanasuppat, K., Wongkham, W., Meepowpan, P., et al., (2009). In vitro screening for anthelmintic and antitumour activity of ethnomedicinal plants from Thailand. J. Ethnopharmacol. 123, 475- 482.
15. Masuma, R., Shiomi, K., Omura, S., (2009). Helminth electron transport inhibitors produced by fungi. In: Anke, T., Weber, D. 1st Edition. Physiology and Genetics. Springer Berlin Heidelberg, pp. 247-271.

16. Zhang, S. M., Coultas, K. A. (2013). Identification of plumbagin and sanguinarine as effective chemotherapeutic agents for treatment of schistosomiasis. Int. J. ParasitolDrug. 3, 28-34.

17. Makwali, J.A., Wanjala, F.M.E., Ingonga, J., Anjili, C.O., (2015). In vitro Studies on the Antileishmanial Activity of Herbicides and Plant Extracts Against Leishmania major Parasites. Research Journal of Medicinal Plants. 9,90-104.

18. Gupta, A.C., Mohanty, S., Saxena, A., Maurya, A.K., Bawankule, D.U., (2018). Plumbagin, a vitamin K3 analogue ameliorate malaria pathogenesis by inhibiting oxidative stress and inflammation. Inflammopharmacology. 26, 983-991.

19. Babula, P., Adam, V., Havel, L., Kizek, R., (2007). Naftochinony a jejich farmakologické vlastnosti [Naphthoquinones and their pharmacological properties]. Ceska. Slov. Farm. 56,114-120.

20. Sumsakul, W., Plengsuriyakarn, T., Chaijaroenkul, W., Viyanant, V., Karbwang, J., Na- Bangchang, K., (2014). Antimalarial activity of plumbagin in vitro and in animal models. BMC Complem. Altern. M. 14,15 .

21. Saowakon, N., Tansatit, T., Wanichanon, C., Chanakul, W., Reutrakul, V., Sobhon, P. (2009). 
Fasciola gigantica: anthelmintic effect of the aqueous extract of Artocarpus lakoocha. Exp. Parasitol. 122, 289-98.

22. Shalaby, H. A., El Namaky, A. H., Kamel, R. O., (2016). In vitro tegumental alterations on adult Fasciola gigantica caused by mefloquine. J. Parasit. Dis.40, 145151.

23. Dangprasert, T., Khawsuk, W., Meepool, A., et al., (2001). Fasciola gigantica: Surface topography of the adult tegument. Journal of helminthology. 75, 43-50. Brophy, P.M., Barrett, J., 1990. Glutathione transferase in helminths. Parasitology. 100, 345-349.

24. Alvarez-Mercado, J.M., IbarraVelarde, F., Alonso-Díaz, M.Á. VeraMontenegro, Y., Avila-Acevedo, J.G, García-Bores A.M., (2015). In vitro antihelmintic effect of fifteen tropical plant extracts on excysted flukes of Fasciola hepatica. BMC Vet. Res. 11, 45.

25. McCarthy J.S., Moore, T. A., 2015. 42 - Drugs for Helminths, Editor(s): John E. Bennett, Raphael Dolin, Martin J. Blaser. In: Mandell, Douglas, and Bennett's Principles and Practice of Infectious Diseases. $8^{\text {th }} \mathrm{Edition}$. Content Repository Only! pp 519527.e3.

26. Toner, E., Brennan, G.P., Hanna, R.E., Edgar, H.W., Fairweather, I., (2010). Time-dependent changes to the tegumental system and gastrodermis of adult Fasciola hepatica following treatment in vivo with triclabendazole in the sheep host. Vet. Parasitol. 174:218-2 27.
27. Sachanonta, N., Chotivanich, K., Chaisri, U., et al., (2011). Ultrastructural and real-time microscopic changes in $P$. falciparum-infected red blood cells following treatment with antimalarial drugs. Ultrastruct. Pathol. 35, 214225.

28. Massoud, A. M., Shalaby, H. A., El Khateeb, R. M., Mahmoud, M. S., Kutkat, M. A., (2013). Tegumental histological effects of Mirazid(®) and myrrh volatile oil on adult Fasciola gigantica. Asian Pac. J. Trop. Biomed. 3, 501-504.

29. Lorsuwannarat N, Piedrafita D, Chantree P, et al., (2014). The in vitro anthelmintic effects of plumbagin on newly excysted and 4-weeks- old juvenile parasites of Fasciola gigantica. Exp. Parasitol. 136,5-13.

30. Keiser, J., Morson, G. (2008). Fasciola hepatica: surface tegumental responses to in vitro and in vivo treatment with the experimental fasciolicide OZ 78. Exp. Parasitol. 119, 87-93.

31. Shalaby, H.A., El Namaky, A.H., Kamel, R.O.A., 2009. In vitro effect of artemether and triclabendazole on adult Fasciola gigantica. Vet. Parasitol. 160,76-82.

32. Shehab, N.G., Monem, A.R., Hassan, S.E., Toaleb, N.I., (2009). Botanical study of Meryta denhamii Seem. and its antihelminthic activity against Fasciola gigantica. J. Egypt Soc. Parasitol. 39, 269-288.

33. Lorsuwannarata, N., Saowakonb, N. Ramasootac, P., Wanichanona, C., Sobhona P., (2013). The anthelmintic effect of plumbagin on Schistosoma 
mansoni Exp. Parasitol. 133, 18- 27.

34. Awasthi, B.P., Kathuria, M., Pant, G., Kumari, N., Mitra, K., (2016). Plumbagin, a plant- derived naphthoquinone metabolite induces mitochondria mediated apoptosis-like cell death in Leishmania donovani: an ultrastructural and physiological study. Apoptosis. 21, 941-953. 